\title{
Real-world experience of SARS-CoV-2 antibody assays in UK healthcare workers
}

\author{
Authors: Alyss V Robinson, ${ }^{A}$ Gary Weaving, ${ }^{\mathrm{B}}$ Barbara J Philips, ${ }^{\mathrm{C}}$ Alice C Eziefula, ${ }^{\mathrm{D}}$ Kate E Shipman ${ }^{\mathrm{E}}$ and \\ Timothy Chevassut ${ }^{F}$
}

\section{Background}

The seroprevalence of antibodies to SARS-CoV-2 in healthcare workers is variable throughout the world. This study compares the use of two antibody assays among large cohorts of healthcare workers in southern England.

\section{Methods}

This cohort study includes data obtained from staff at Western Sussex Hospitals NHS Foundation Trust (WSHT) and Brighton and Sussex University Hospitals (BSUH) during voluntary antibody testing, using Abbott and Roche SARSCoV-2 antibody assays at each Trust respectively.

Results

The observed seroprevalence level was $7.9 \%$ for the WSHT/ Abbott cohort versus $13 \%$ for the BSUH/Roche cohort. Based on a previous positive $\mathrm{PCR}$, we find that the false-negative rate of the Abbott and Roche assays were $60.2 \%$ and $19 \%$ respectively, implying sensitivity levels of $39.8 \%$ and $81 \%$. Within these cohorts, seropositivity was most strongly associated with those of South Asian ethnicity, allied health professionals and male $\operatorname{sex}(p<0.0001)$.

Conclusions

In this real-world study, neither antibody test performed to the specification level stated by the manufacturer. More rigorous testing of these and other assays in target populations is recommended prior to widespread usage if they are to provide data that might be useful to control the pandemic.

Authors: ${ }^{A}$ academic foundation trainee, Brighton and Sussex University Hospitals NHS Trust, Brighton, UK; ${ }^{B}$ senior clinical scientist, Brighton and Sussex University Hospitals NHS Trust, Brighton, UK; Creader, Brighton and Sussex Medical School, University of Sussex, Brighton, UK, and consultant in intensive care medicine, Brighton and Sussex University Hospitals NHS Trust, Brighton, UK; Dsenior lecturer, Brighton and Sussex Medical School, University of Sussex, Brighton, UK, and consultant in infectious disease, Brighton and Sussex University Hospitals NHS Trust, Brighton, UK; ${ }^{E}$ consultant in chemical pathology, Western Sussex Hospitals NHS Foundation Trust, Worthing, UK; Freader, Brighton and Sussex Medical School, University of Sussex, Brighton, UK, and consultant in haematology, Brighton and Sussex University Hospitals NHS Trust, Brighton, UK
KEYWORDS: SARS-CoV-2, seroprevalence, healthcare workers, COVID-19, antibody assay

DOI: $10.7861 /$ clinmed.2020.1007

\section{Summary}

What is known?

There are a variety of different antibody tests for the detection of previous SARS-CoV-2 infection, which were widely used to determine seroprevalence among healthcare workers and the general population. Concerns were raised as these antibody assays were not validated in such populations and may not produce meaningful results.

\section{What is the question?}

What is the seroprevalence of SARS-CoV-2 antibodies among two cohorts of healthcare workers and how have two different antibody assays, Abbott and Roche, performed within these cohorts?

What was found?

The seroprevalence of two similar UK regions was 7.9\% using the Abbott and $13 \%$ using the Roche assay. The real-world sensitivities were only $39.8 \%$ and $81 \%$ respectively. Male sex, South Asian ethnicity and healthcare assistants were most strongly associated with antibody positivity.

What is the implication for practice now? Real-world data suggests antibody assays do not perform to manufacturers' specifications when used on healthcare workers. Side-by-side analysis of different assays within their target populations may be necessary to ensure valid and meaningful results.

\section{Introduction}

The SARS-CoV-2 global pandemic has demanded rapid mobilisation of healthcare resources and unprecedented public health interventions. The UK has now surpassed three million cases and 90,000 deaths.

Antibody testing for SARS-CoV-2 has been a major component of public health campaigns worldwide. ${ }^{2,3}$ However, concerns have been raised about the rapid uptake of testing, questioning the 


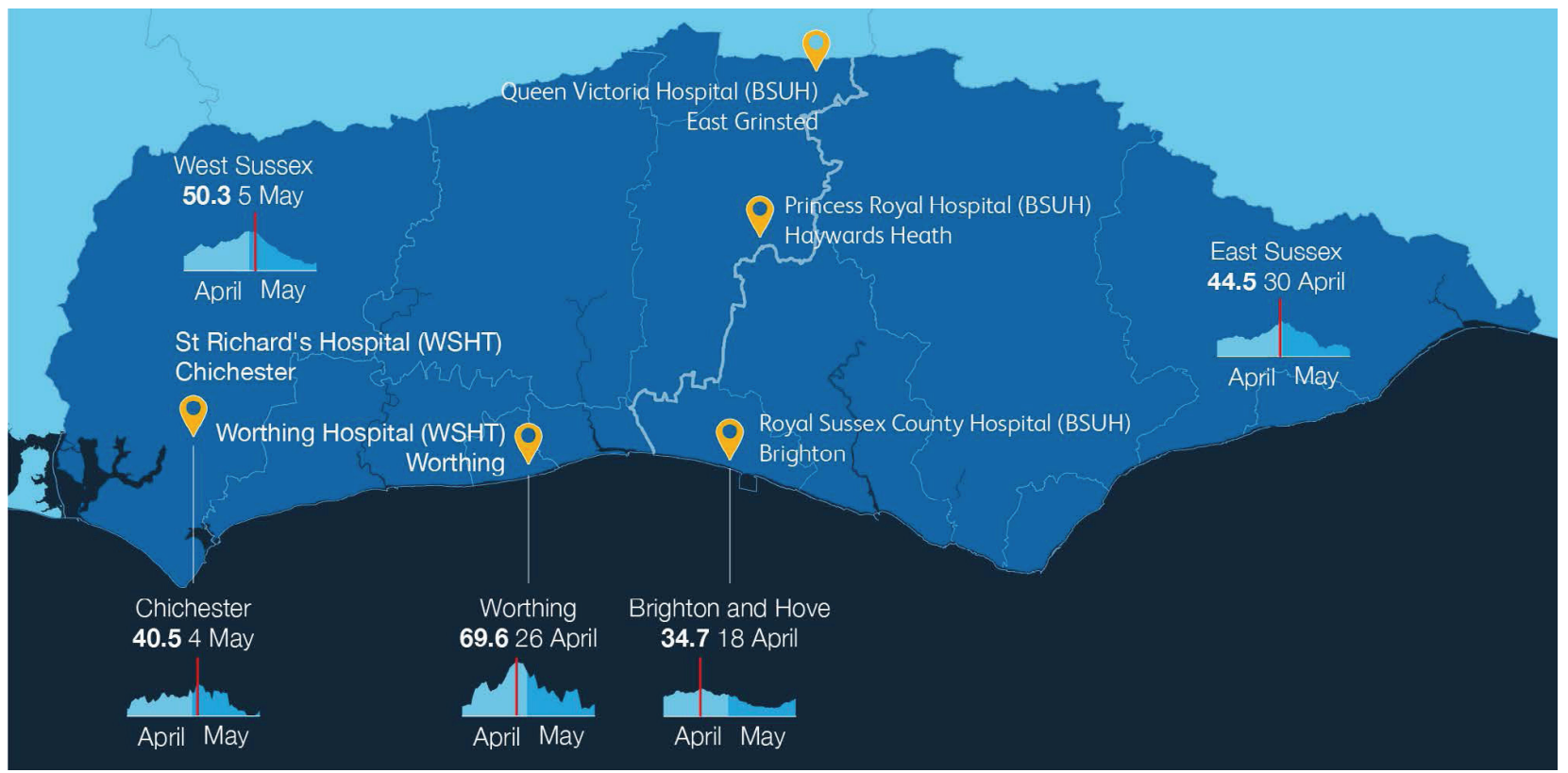

Fig 1. Epidemiological differences across the region served by Western Sussex Hospitals NHS Foundation Trust (WSHT) and Brighton and Sussex University Hospitals (BSUH). Data from Public Health England.

relevance and also the validity of tests available within the target populations. ${ }^{4,5}$ Further, the interpretation of results is limited as the presence of antibodies does not guarantee immunity to future infection. ${ }^{6}$

In the UK, serum antibody testing was introduced and offered to almost all NHS staff from 25 May 2020.7 Global disparities in seroprevalence among healthcare workers have been seen ${ }^{8-13}$ and attributed to local incidence, personal protective equipment (PPE) availability and hospital organisation. ${ }^{14,15}$ Published and in-press data highlight significant disparities in seroprevalence among differing ages, ethnicities, occupations, workplaces and geographical areas, with some of the highest rates recorded in the UK. 8,12,15 However, differences in assay may also be a contributor and this has yet to be appreciated in a real-world dataset.

Western Sussex Hospitals NHS Foundation Trust (WSHT) and Brighton and Sussex University Hospitals (BSUH) worked collaboratively under the same management during the first wave of the pandemic. Epidemiological differences across the region are represented in Fig 1 (data from Public Health England [PHE]1). It can be appreciated that during the first wave of the pandemic, infection rates were higher in WSHT regions than BSUH. The estimated general seroprevalence of the South East is $4.7 \%{ }^{16}$

PHE have now evaluated eight different antibody assays, the first of which were provided by Abbott (for which PHE report a sensitivity of $92.7 \%$ and specificity of $100 \%{ }^{17}$ ) and Roche (sensitivity of $83.9 \%$ and specificity of $100 \%{ }^{18}$ ). In line with centralised allocation of testing platforms and local availability of analysers, WSHT used the Abbott assay and BSUH used the Roche assay for staff antibody testing.

The aim of this study was to determine the seroprevalence of SARS-CoV-2 antibodies among healthcare workers in these regions and compare the performance of the different antibody assays within their respective cohorts.

\section{Methods \\ Participants}

This retrospective cohort study is written in accordance with the STROBE ${ }^{19}$ and STARD ${ }^{20}$ guidance. All antibody, SARS-CoV-2 polymerase chain reaction (PCR) viral nasopharyngeal throat swab and survey data from staff offered SARS-CoV-2 antibody testing via BSUH and WSHT were included. Anonymised data were obtained from the prospectively recorded results databases at each hospital. The survey proforma differed at each trust, but included age, test date, presence of symptoms (binary), symptom onset date, antibody titre and the presence of a positive PCR at both sites. BSUH also included source of test (acute care hospital, primary care, community services, mental health services etc), and date of PCR. The WSHT dataset included ethnicity and profession. The PCR results at WSHT were grouped into positive or negative/ not taken, whereas at BSUH these were either positive, negative or not taken.

Staff were invited for voluntary antibody testing from the 19 May 2020 onwards. The database was censored as of 29 September 2020 for BSUH and 20 October 2020 for WSHT.

\section{Materials}

Reverse-transcription polymerase chain reaction (PCR) nasopharyngeal swab sampling was used to confirm SARS-CoV-2 infection in upper respiratory specimens for staff with symptoms of COVID-19.

At WSHT, staff blood antibody samples were collected in BD Vacutainer serum separator tubes ${ }^{\circledR}$, spun on arrival and analysed according to manufacturer instruction using the Abbott ARCHITECT i2000 (Abbott, California). The Abbott assay is a two-step chemiluminescent microparticle immunoassay (CMIA) for detection of IgG to SARS-CoV-2 nucleocapsid protein. At BSUH, samples were collected using BD Vacutainers ${ }^{\circledR}$ and EDTA 
Vacuettes $^{\circledR}$ (Greiner Bio-One) and analysed using the Cobas e411 analyser (Roche Diagnostics, Mannheim Germany) and Roche Elecsys ${ }^{\circledR}$ Anti-SARS-CoV-2 sandwich immunoassay. Roche Elecsys ${ }^{\circledR}$ is an antigen-based electrochemiluminescent immunoassay (ECLIA) designed to detect IgM and IgG antibodies to SARS-CoV-2 nucleocapsid protein using biotinylated antigen and antigen labelled with ruthenium.

The manufacturer-reported sensitivity and specificity is $100 \%$ and $99.6 \%$ at 14 days for Abbott, and $99.5 \%$ and $99.8 \%$ for Roche. A positive antibody serology is defined by a relative light unit (RLU) above 1.4 for Abbott and a cut of index (COI) greater than 1.0 for the Roche assay.

\section{Statistical analysis}

Data were analysed in IBM SPSS version 26. Between-group comparisons were made using the independent samples t-test for parametric data, Mann-Whitney U test for non-parametric data and Chi-squared or Fisher's exact test for proportions where appropriate. Risk factors for antibody positivity were determined using univariate binary logistic regression.

A positive PCR prior to antibody testing was considered the 'gold standard' for confirmed SARS-CoV-2 infection. Missing data were excluded per analysis. There is no a-priori sample size calculation for the study as we have used all available data at both institutions.

\section{Ethics}

The study was carried out as a test validation of new antibody assays. The analysis was carried out on fully anonymised and non-identifiable data.

\section{Results}

Overall, 26,861 SARS-CoV-2 antibody tests were processed across both sites over a 4-month period: 12,388 at WSHT and 14,473 at BSUH. Positive assays were present in 978 (7.9\%) WSHT staff and $1,880(13 \%)$ at BSUH. Positive PCR results were recorded in 566 staff members at WSHT and 163 staff members at BSUH prior to antibody testing, giving an overall known infection rate of $4.9 \%$ and $1.1 \%$ at each site respectively.

Antibody tests occurred a median 97 days after symptoms onset in those with a positive PCR in the WSHT cohort, and 53 days after PCR and 61 days after symptom onset in the BSUH cohort. Symptom reporting was marginally higher at BSUH than WSHT ( $29.6 \%$ versus $27.6 \%$ respectively). The rates of antibody detection in asymptomatic staff were $3.3 \%$ for WSHT and $6.9 \%$ for BSUH (Table 1). When comparing the antibody results to a prior positive PCR result (ie the current gold standard test for infection with SARS-CoV-2), only $39.8 \%$ of staff at WSHT and $81 \%$ of staff at BSUH demonstrated a positive antibody assay. In staff at WSHT who both experienced symptoms and demonstrated a positive PCR result $(n=362)$, only $54.7 \%$ demonstrated antibodies. At BSUH, only two cases with symptoms and a positive PCR had a negative antibody test, raising the sensitivity to $87.5 \%$; however, the numbers in this subset at BSUH were very small.

Of cases with a negative antibody test at WSHT, the RLUs were not significantly different in those with or without a positive PCR (median 0.2 in both groups, $p=0.102$ ). Similarly, at BSUH the COI was comparable (median 0.09 in both groups, $p=0.162$ ). This
Table 1. Antibody test performance based on symptoms and PCR results

Antibody result

Not detected Detected p-value

WSHT/Abbott cohort

Symptoms

No

Yes

$8,113(96.7 \%) \quad 276(3.3 \%)$

$<0.0001$

PCR

Negative/not taken

$2,578(80.9 \%) \quad 610(19.1 \%)$

Positive

$10,294(94.0 \%)$

$655(6.0 \%)$

$<0.0001$

BSUH/Roche cohort

Symptoms

No

$9,485(93.1 \%)$

$703(6.9 \%)$

$<0.0001$

Yes

$3,108(72.5 \%) \quad 1,178(27.5 \%)$

PCR

$\begin{array}{llll}\text { Negative } & 79(70.5 \%) & 33(29.5 \%) & <0.0001 \\ \text { Positive } & 4(19 \%) & 17(81 \%)\end{array}$

BSUH = Brighton and Sussex University Hospitals; $\mathrm{PCR}=$ polymerase chain reaction; WSHT $=$ Western Sussex Hospitals NHS Foundation Trust

demonstrates that the false negatives did not have a significantly higher raw RLU or COI in either cohort than the true negatives. Of those with a positive antibody result, the RLUs were significantly higher in those with a positive PCR, compared with a negative, at WSHT (median 4.86 versus 4.05, $p<0.0001$ ). The same was not true at BSUH ( 41.7 for positive PCR versus 60.7 for negative PCR, $\mathrm{p}=0.213$ ).

In the WSHT cohort, antibodies were detected up to 213 days after symptom onset with a median of 135 days (interquartile range [IQR] 126-168). By contrast, in the BSUH cohort, antibodies were detected in PCR-proven individuals up to 134 IQR test, with a median of 91 days (IQR 73-113) and in those with symptoms without a PCR test up to 195 days post-symptom onset.

Factors significantly associated with antibody positivity are described in Table 2. Male sex was associated with an increased rate of seropositivity by approximately $30 \%$. While age was a significant finding, the overall effect size is negligible. South Asian ethnicity demonstrated in the order of three times the risk of seropositivity, with Black and East Asian ethnicity also conferring an increased risk. The 'medical' occupation category had the highest proportion of South Asian (8.8\%), and Black staff (2.8\%). Allied health professionals and nursing staff were high-risk, with medical staff being the lowest risk of front-line workers. The main hospital and local hospice had far higher risk of seropositivity than other workplace categories. Community, mental health, general practice and staff working in an elective surgical facility had a comparably low association with antibody positivity.

\section{Discussion}

This study provides real-world data on the efficacy of both Abbott and Roche SARS-CoV-2 antibody assays. Despite the higher incidence within the WSHT cohort of PCR-proven COVID-19 
Table 2. Univariate analysis of risk factors for seropositivity

\begin{tabular}{|c|c|c|}
\hline & Odds ratio $(95 \% \mathrm{CI})$ & $p$-value \\
\hline Male sex & $1.36(1.24-1.49)$ & $<0.0001$ \\
\hline \multicolumn{3}{|l|}{ Ethnicity (WSHT data) } \\
\hline Caucasian & Reference & \\
\hline Black & $2.18(1.35-3.51)$ & 0.001 \\
\hline East Asian & $2.39(1.70-3.36)$ & $<0.0001$ \\
\hline South Asian & $3.03(2.25-4.10)$ & $<0.0001$ \\
\hline \multicolumn{3}{|c|}{ Occupation (WSHT data) } \\
\hline Administrative & Reference & \\
\hline Medical & $2.41(1.67-3.49)$ & $<0.0001$ \\
\hline Porter & $2.68(1.33-5.40)$ & $<0.0001$ \\
\hline Paramedic & $2.97(1.93-4.57)$ & $<0.0001$ \\
\hline Nurse & $3.03(2.15-4.27)$ & $<0.0001$ \\
\hline $\begin{array}{l}\text { Allied health/healthcare } \\
\text { assistants }\end{array}$ & $3.78(2.63-5.43)$ & $<0.0001$ \\
\hline \multicolumn{3}{|l|}{ Workplace (BSUH data) } \\
\hline Elective surgery facility & Reference & \\
\hline Care homes & $2.30(1.58-3.34)$ & $<0.0001$ \\
\hline Ambulance service & $2.73(1.95-3.81)$ & $<0.0001$ \\
\hline Acute care hospitals & $3.15(2.31-4.28)$ & $<0.0001$ \\
\hline Hospice & 3.61 (1.89-6.94) & $<0.0001$ \\
\hline
\end{tabular}

BSUH = Brighton and Sussex University Hospitals; $\mathrm{CI}=$ confidence interval; WSHT $=$ Western Sussex Hospitals NHS Foundation Trust

infections, a higher case rate in the region served by WSHT (Fig 1), and similar symptom reporting, a far lower incidence of antibody positivity is seen at WSHT (7.9\% versus $13 \%)$. Notably, the majority of staff who had a prior positive PCR with Abbott actually had a negative antibody test (60.2\%). This figure was slightly improved in those who also reported symptoms, with $54.7 \%$ demonstrating antibodies at WSHT and $87.5 \%$ at BSUH.

The RLUs and COI for Abbott and Roche were not significantly different for true negatives versus false negatives, so the chosen threshold was unlikely to have contributed significantly to the sensitivity of the tests. The RLUs were significantly higher for the Abbott test in those with a PCR-proven infection. However, this was not observed with the Roche assay and there are a number of confounding factors which may influence this observation so this must be interpreted with caution. There is no convincing literature to suggest that either assays are quantitative.

There is a growing collection of published healthcare worker antibody data from across the world, with a wide range of seropositivity, from $<1 \%$ in Japan, ${ }^{13,21}<3 \%$ in Germany ${ }^{22}$ to up to $30 \%$ in the UK, $, 810,12$ Pakistan, ${ }^{9}$ and Sweden. ${ }^{11}$ However, the large disparity between two NHS trusts under the same management and serving the same region of the UK was unexpected. The overall differences in seroprevalence between the test sites in this study may be due to differences in hospital structure, provision of PPE, caseload and availability of PCR testing for staff. However, the false-negative rate in both cohorts and the higher burden of disease in the WSHT region raises the suspicion that the overall seroprevalence may have been under-estimated, and most significantly so in the Abbott assay cohort.

Test performance of antibody assays were determined and validated using symptomatic, hospitalised patients. ${ }^{23} \mathrm{~A}$ metaanalysis of diagnostic accuracy tests for SARS-CoV-2 serology found sensitivities ranging from $14.4-100 \%$ depending on serological test method and immunoglobulin class. ${ }^{24} \mathrm{~A}$ general population study of 1,862 people in Austria found IgA antibodies in $11 \%$ whereas IgG in only $1.9 \% .{ }^{25}$ Discordance between assays has been reported, ${ }^{26}$ and one UK study found that $58 \%$ of Abbottnegative samples demonstrated other SARS-CoV- 2 antibodies. ${ }^{27}$

The generalisability of antibody testing to healthcare workers with variable viral exposure (and presumably low overall seroprevalence) remains unknown, despite many thousands having been undertaken in the UK to-date. Therefore, the mass testing of healthcare workers has been controversial. ${ }^{4,5}$ In this cohort, antibody tests were performed 61 days at BSUH and 97 days at WSHT postsymptom onset. More recent data have emerged which suggest that antibody responses in mildly symptomatic or asymptomatic individuals decline after 1 month, ${ }^{28}$ and therefore within both cohorts the window for serological testing may have been missed. However, the evidence is conflicting, and studies have seen individuals with persistent antibodies beyond 6 months. ${ }^{29}$ As those with a positive PCR in this study represent symptomatic individuals, even if some cases were beyond the window for when IgG would still be detectable it would be expected to be greater than $40 \%$ in any case. We saw cases with positive antibodies up to 213 days post-symptom onset. Importantly, it is clear that not all individuals will seroconvert despite confirmed SARS-CoV-2 infection, with younger age and less severe infection being associated with a lower probability of seroconversion. This represents many healthcare staff. Therefore, whether current SARS-CoV-2 antibody assays can provide reliable epidemiological data is largely undetermined.

During the pandemic, finances, resources and supply chains are precious. In the UK, mass-vaccination programmes, general population antibody tests using the lateral flow immunoassay $(\text { LFIA })^{30}$ and lateral flow antigen assays for healthcare workers are underway. The SIREN study aims to serially test 100,000 healthcare workers across the UK to monitor antibodies over time. Therefore, there is unmet need for high-quality side-by-side assessment of antibody and antigen tests in the real-world environment in order to capture accurate epidemiology and vaccine response. Of note, neither the Abbott nor Roche tests will identify antibody responses to the Pfizer-BioNTech and Oxford-AstraZeneca vaccines that use the spike protein to generate immunity.

Further, in December 2020, a new and highly infectious variant of SARS-CoV-2 was identified in an individual in the UK with antibodies to SARS-CoV-2, 8 months after initial infection. ${ }^{31} \mathrm{~A}$ number of further mutant variants have been identified, which questions the protection from early SARS-CoV-2 antibodies. At this stage, it is not clear whether prior SARS-CoV-2 infection is protective against the new variant strains despite reassurances that the current vaccines should remain efficacious. Data on the longevity and waning of antibodies are emerging but often conflicted. With the rollout of vaccination programmes, and in the face of emerging variants, there is an urgent need to better understand the kinetics of antibody responses against SARS-CoV-2.

We also observed significant differences in workplace, occupation and ethnicity and risk of seropositivity. Given that the majority of Black and South Asian staff were in the medical 
profession (which was a lower risk staff category), the increased risk of antibodies is particularly striking. The reasons for this are unclear, and demand investigation, as severity of illness and risk of death is also greater in Black and ethnic minority individuals. ${ }^{32}$

\section{Limitations}

Using a previous positive PCR result to determine the efficacy of the antibody test has a number of limitations. While there may be false positivity of the PCR, the risk is mitigated in this cohort as during the first wave of the pandemic only symptomatic individuals were invited for PCR testing. Nevertheless, it would be expected that the vast majority of, if not all, positive PCR cases would have antibodies present on serology; thus the sensitivity findings provide a reasonable estimate of real-world performance. However, we have chosen not to comment on specificity for a number of reasons; the suboptimal sensitivity of the PCR (approximately $73.3 \%{ }^{33}$ ), the fact that those with a negative PCR and the timing of PCR was not specified in the WSHT cohort, the absence of PCR testing for staff early in the pandemic and the fact that some cases are asymptomatic.

The requirement to carry out large-scale serological testing on staff at short notice meant that data collection was inconsistent across sites, leading to missing data. Consequently, important characteristics such as working environment, PPE use and specific symptomatology were not captured. Not all staff received an antibody test as this was voluntary, which may have introduced selection bias. We do not know the overall rates of uptake.

\section{Conclusion}

Serum antibody tests on healthcare workers may not accurately reflect the seroprevalence in this population and are likely to have underestimated the true incidence of SARS-CoV-2 infections. In our real-world dataset presented here, we find the two most widely used antibody tests in the UK, Roche and Abbott, have a real-world sensitivity level of $81 \%$ and $39.8 \%$ respectively. More research, based on strict criteria of appropriate timing and indication, is urgently required to establish the true validity of different SARSCoV-2 antibody assays in real world settings.

\section{Acknowledgements}

We would like to acknowledge Sarah Fitzpatrick, graphic designer, for her production of Fig 1.

\section{References}

1 Public Health England and NHSX. Coronavirus (COVID-19) in the UK. PHE, 2020. https://coronavirus.data.gov.uk [Accessed 1 November 2020].

2 Peeling RW, Wedderburn C], Garcia PJ et al. Serology testing in the COVID-19 pandemic response. Lancet Infec Dis 2020;9:e245-9.

3 Department of Health and Social Care. Coronavirus (COVID-19): Scaling up our testing programmes. DHSC, 2020. https://assets. publishing.service.gov.uk/government/uploads/system/uploads/ attachment_data/file/878121/coronavirus-covid-19-testingstrategy.pdf [Accessed 4 November 2020].

4 Andersson M, Low N, French N et al. Rapid roll out of SARS-CoV-2 antibody testing - a concern. BMJ 2020;369:m2420.

5 Armstrong S. Why covid-19 antibody tests are not the game changer the UK government claims. BMJ 2020;369:m2469.
6 Huang A, Garcia-Carreras B, Hitchings MDT et al. A systematic review of antibody mediated immunity to coronaviruses: antibody kinetics, correlates of protection, and association of antibody responses with severity of disease. Nat Commun 2020;11:4704.

7 Philip P, Powis S. Antibody testing programme roll out for NHS staff and patients. NHS England, 2020. www.england.nhs.uk/coronavirus/wp-content/uploads/sites/52/2020/05/antibody-testingprogramme-letter-25-may-2020.pdf [Accessed 30 October 2020].

8 Grant J], Wilmore SMS, McCann NS et al. Seroprevalence of SARS-CoV-2 antibodies in healthcare workers at a London NHS Trust. Infect Control Hosp Epidemiol 2021;42:212-4.

9 Haq M, Rehman A, Noor M et al. Seroprevalence and risk factors of SARS CoV-2 in health care workers of tertiary-care hospitals in the province of Khyber Pakhtunkhwa, Pakistan. medRxiv 2020, 2020.09.29.20203125.

10 Shields A, Faustini SE, Perez-Toledo M et al. SARS-CoV-2 seroprevalence and asymptomatic viral carriage in healthcare workers: A cross-sectional study. Thorax 2020;75:1089-94.

11 Lindahl JF, Hoffman T, Esmaeilzadeh M et al. High seroprevalence of SARS-CoV-2 in elderly care employees in Sweden. Infect Ecol Epidemiol 2020;10:1789036.

12 Favara DM, McAdam K, Cooke A et al. SARS-CoV-2 antigen and antibody prevalence among UK staff working with cancer patients during the COVID-19 pandemic. medRxiv 2020; 2020.09.18.20197590.

13 Fukuda H, Seyama K, Ito K et al. SARS-CoV-2 seroprevalence in healthcare workers at a frontline hospital in Tokyo. Res Sq 2020, in press (doi: 10.21203/rs.3.rs-96870/v1).

14 Poulikakos D, Sinha S, Kalra PA. SARS-CoV-2 antibody screening in healthcare workers in a tertiary centre in North West England. $]$ Clin Virol 2020;129:104545.

15 Lai CC, Wang JH, Hsueh PR. Population-based seroprevalence surveys of anti-SARS-CoV-2 antibody: An up-to-date review. Int J Infect Dis 2020;101:314-22.

16 Public Health England. Sero-surveillance of COVID-19. www.gov.uk/ government/publications/national-covid-19-surveillance-reports/ sero-surveillance-of-covid-19 [Accessed 25 November 2020].

17 Public Health England. Evaluation of the Abbott SARS-CoV-2 IgG for the detection of anti-SARS-CoV-2 antibodies. PHE, 2020. https://assets.publishing.service.gov.uk/government/uploads/ system/uploads/attachment_data/file/890566/Evaluation_of_ Abbott_SARS_CoV_2_IgG_PHE.pdf [Accessed 15 October 2020].

18 Public Health England. Evaluation of Roche Elecsys AntiSARS-CoV-2 serology assay for the detection of anti-SARS-CoV-2 antibodies. PHE, 2020. https://assets.publishing.service.gov.uk/ government/uploads/system/uploads/attachment_data/file/ 891598/Evaluation_of_Roche_Elecsys_anti_SARS_CoV_2 PHE_200610_v8.1_FINAL.pdf [Accessed 15 October 2020].

19 von Elm E, Altman DG, Egger $M$ et al. The strengthening and reporting of observational studies in epidemiology (STROBE) statement: guidelines for reporting observational studies. J Clin Epidemiol 2008:61:344-9.

20 Bossuyt PM, Reitsma JB, Bruns DE et al. STARD 2015: an updated list of essential items for reporting diagnostic accuracy studies. BMJ 2015;28:h5527.

21 Nishida T, Iwahashi H, Yamauchi K et al. Seroprevalence of SARS-CoV-2 antibodies among 925 staff members in an urban hospital accepting COVID-19 patients in Osaka Prefecture, Japan. SSRN Electron J, in press (doi: 10.2139/ssrn.3670675).

22 Erber J, Kappler V, Haller B et al. Strategies for infection control and prevalence of anti-SARS-CoV-2 IgG antibodies in 4,554 employees of a university hospital in Munich, Germany. SSRN Electron J, in press (doi: 10.2139/ssrn.3678599).

23 Deeks J], Dinnes J, Takwoingi Y et al. Antibody tests for identification of current and past infection with SARS-CoV-2. Cochrane Database Syst Rev 2020;6:CD013652. 
24 Lisboa Bastos M, Tavaziva G, Abidi SK et al. Diagnostic accuracy of serological tests for covid-19: Systematic review and meta-analysis. BMJ 2020;370:m2516.

25 Knabl L, Mitra T, Kimpel ] et al. High SARS-CoV-2 seroprevalence in children and adults in the Austrian ski resort Ischgl. medRxiv 2020; 10.1101/2020.08.20.20178533.

26 Perkmann T, Permann-Nagele N, Breyer MK et al. Side by side comparison of three fully automated SARS-CoV-2 antibody assays with a focus on specificity. Clin Chem 2020;66:1405-13.

27 Rosadas C, Randell P, Khan M et al. Testing for responses to the wrong SARS-CoV-2 antigen? Lancet 2020;396:e23.

28 Röltgen K, Powell AE, Wirz OF et al. Defining the features and duration of antibody responses to SARS-CoV-2 infection associated with disease severity and outcome. Sci Immunol 2020;5:eabe0240.

29 Choe PG, Kang CK, Suh H] et al. Waning antibody responses in asymptomatic and symptomatic SARS-CoV-2 infection. Emerg Infect Dis 2021;27:327-9.
30 Ward $\mathrm{H}$, Atchison C], Whitaker $\mathrm{M}$ et al. Antibody prevalence for SARS-CoV-2 in England following first peak of the pandemic: REACT2 study in 100,000 adults. medRxiv 2020; 10.1101/2020.08.12.20173690.

31 Harrington D, Kele B, Pereira S et al. Confirmed reinfection with SARS-CoV-2 variant VOC-202012/01. Clin Infect Dis 2021, in press (doi: 10.1093/cid/ciab014).

32 Pareek M, Bangash MN, Pareek N et al. Ethnicity and COVID-19: an urgent public health research priority. Lancet 2020;395:1421-2.

33 Böger B, Fachi MM, Vilhena RO et al. Systematic review with meta-analysis of the accuracy of diagnostic tests for COVID-19. Am J Infect Control 2020;49:21-9.

Address for correspondence: Alyss V Robinson, Royal Sussex County Hospital, Brighton BN2 5BE.

Email: alyss.robinson@nhs.net 